\title{
EDITORIAL
}

\section{WOULD TAMING THE T-CELL QUIETEN THE EYE?}

Immunological dysfunction can cause a great many problems in the eye, most of which are, at best, only partially amenable to current therapy. Almost any ocular structure can be involved, from the fascia and muscles of the orbit, through the coats and contents of the globe, to the conjunctiva and cornea. The severity of the ensuing damage can vary from tissue destruction in necrotising scleritis, to the inflammatory dysfunction of optic neuritis and posterior uveitis, or the delayed reparative sequelae of fibrosis and scarring, well seen in the conjunctiva in cicatrising pemphigoid and the ocular muscles in dysthyroid eye disease.

Surgical progress may also be seriously compromised by the response of the immune system, the best known example being corneal graft rejection following transplantation. Furthermore, inflammatory and immune responses are thought to contribute to the development of proliferative vitreoretinopathy (PVR) after retinal detachment and its attendant surgery.

Orbital and ocular tumours may also be influenced by the immune response, since histologic examination often demonstrates lymphocytes surrounding or infiltrating tumour tissue, thus potentially regulating or modifying tumour development and behaviour.

In all the above ocular disorders, the villains are considered to be $T$ lymphocytes, and these cells can have a variety of effects in differing situations. Two major subsets of $\mathrm{T}$ lymphocytes are now recognised by the expression of specific markers (CD4 or CD8) on the cell surface. $\mathrm{CD} 4^{+} \mathrm{T}$ cells can, for example, secrete soluble factors [lymphokines] which may promote the recruitment of other cells such as macrophages, mast cells and neutrophils into the target tissue. These effector cells may then themselves cause damage to the involved ocular structures. $\mathrm{CD} 4^{+} \mathrm{T}$-cells also cooperate with $\mathrm{B}$ cells to generate antibodies to specific ocular tissue antigens, which can result in cellular damage through fixation of complement. In other situations, $\mathrm{CD} 4^{+} \mathrm{T}$-cells cause cellular damage by secreting other lymphokines such as tumour necrosis factor (TNF $\alpha$ ) which can directly cause cell death. $\mathrm{CD}^{+}$cells are the dominant cells involved in organ-specific autoimmune disease, and are thought to be the cells principally involved in perpetuation mechanisms in conditions such as posterior uveitis, scleritis and dysthyroid eye disease.

$\mathrm{CD}^{+}$cells do, however, also have important beneficial functions. In HIV infection, CMV retinitis occurs with increasing frequency when the $\mathrm{CD} 4^{+}$count drops to low levels, indicating an important role for these cells in combating this retinal infection.

$\mathrm{CD}^{+} \mathrm{T}$-cells can have a direct cytotoxic role and may be involved in corneal graft rejection. They are also known to be important in the immune response against viruses and have been implicated in the pathogenesis of herpetic eye disease. To divide and mature, $\mathrm{CD} 8^{+} \mathrm{T}$-cells normally require lymphokines secreted by activated $\mathrm{CD} 4^{+} \mathrm{T}$-cells, further linking the two subsets.

Thus, the activated T-cell can cause problems for all ophthalmic specialities and, since it cannot yet be surgically removed, further understanding is required before we can thwart its undesirable effects and disrupt its orchestration of serious ocular disease. 